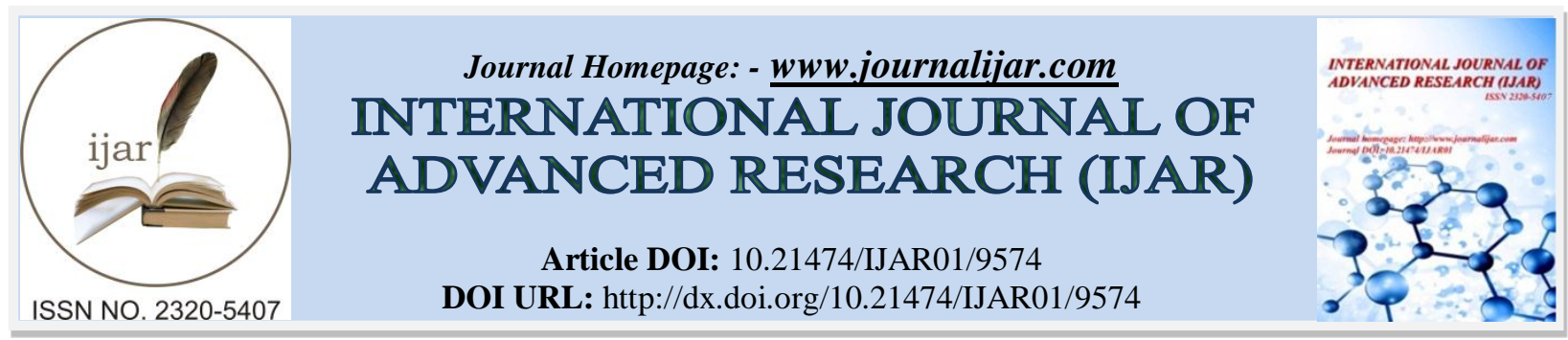

RESEARCH ARTICLE

\title{
A COMPLETE AYURVEDIC REVIEW ON SUNISHANNAKA [MARSILEA QUADRIFOLIA LINN].
}

Jilu Joy $^{1}$, Dr. Sara Monsy Oommen ${ }^{2}$, Dr. Giby Thomas ${ }^{3}$ and Dr. P. Y Ansary ${ }^{4}$.

1. PG Scholar, Dept. of Dravyaguna vijnanam, Govt. Ayurveda College, Tripunithura.

2. Professor, Dept. of Dravyaguna vijnanam, Govt. Ayurveda College, Thiruvananthapuram.

3. Associate Professor, Dept. of Prasooti tantra and Stree roga, Govt. Ayurveda College, Tripunithura.

4. Professor \& HOD, Dept. of Dravyaguna vijnanam, Govt. Ayurveda College, Tripunithura.

\section{Manuscript Info}

Manuscript History

Received: 14 June 2019

Final Accepted: 16 July 2019

Published: August 2019

Key words:-

Ayurvedic review, Sunishannaka,

Marsilea quadrifolia Linn.

\begin{abstract}
Sunishannaka (Marsilea quadrifolia Linn) is a known pteridophytic herb belonging to the family Marsileaceae. Extensive usage of Sunishannaka as an important green leafy vegetable is understood from Samhithas. It is included under pathya saka varga by Acharya Charaka and Vagbhata. Acharya Susrutha has described the drug as a pathyasaka (wholesome leafy vegetable) in Raktapitta disease. In Charaka Samhitha, Arsa roga chikitsa, a Ghruta preparation named Sunishannakachangeri ghruta is mentioned wherein swarasa of the plant is taken, which is indicated in Raktarsa, Tridoshaja raktasrava, Mutragraha, Mandagni, Aruchi, Gudabhramsa etc. Almost all acharyas have highlighted the tridoshahara property of the drug along with grahi, agnikrit, deepana, vrishya etc properties. The article reviews about the categorization, synonyms, karma, doshaghnata, therapeutic indication, amayika prayoga, formulations of Sunishannaka.
\end{abstract}

Copy Right, IJAR, 2019,. All rights reserved.

\section{Introduction:-}

The drug Sunishannaka is botanically correlated as Marsilea quadrifolia Linn belonging to the family Marsileaceae commonly known as European water clover. The Ayurvedic references of the drug can be traced from Charaka samhita, Susruta samhita, Ashtanga hrudaya etc and in almost all Nighantus like Dhanwantari nighantu,Raja Nighantu and Nighantu Ratnakara, Priya nighantu etc. In these Nighantus the drug is indicated for Gulma, Arsha, Meha, Jwara, Swasa, Kushta, Aruchi, Bhrama, Bhranti, Daha etc. The drug is popularly known as 'Neeraral or Nalila thamara' in Kerala. The drug is included under pteridophytes which are primitive vascular plant group. But the drug had been used extensively by the reputed vaidyas in many conditions like uterine bleeding etc.

\subsection{HISTORICAL BACKGROUND}

References of Sunishannaka can be traced from Vedic era itself. In Vaikhanasadharmasutra, mentioning of Sunishannaka among vegetables is seen. ${ }^{[1]}$ Extensive usage of Sunishannaka as an important green leafy vegetable is understood from Samhithas.

Nighantus also gives ample references regarding the plant.

\subsection{ETYMOLOGY[Nirukthi $]^{[2,3]}$ \\ सुष्टु निषण्णं स्वप्नो यस्मात्।}

Corresponding Author:- Jilu Joy.

Address:- PG Scholar, Dept. of Dravyaguna vijnanam, Govt. Ayurveda College, Tripunithura. 
That which produces good sleep.

\section{सुष्टु निषीदन्ति गुणा अस्मिन्।}

The one which possess good qualities.

\subsection{CATEGORIZATION AS PER CLASSICAL TEXT T,5,6,7,8,9,10,11,12,13,14,15,16,17]}

The drug Sunishannaka is classified under various ganas and varga in samhitas and nighantus. These classification is done mainly based on their pharmacological and therapeutic activity.

Table: 1 Classification of Sunishannaka in ancient literature

\begin{tabular}{|c|l|l|}
\hline SI No: & Text & Varga / Gana \\
\hline 1. & Charaka Samhita & Saka varga \\
\hline 2. & Susruta Samhita & Kasaya varga \\
\hline 3. & Ashtanga Hrudaya & Saka varga \\
\hline 4. & Dhanwantari Nighantu & Guducyadi varga \\
\hline 5. & Sodhala Nighantu & Guducyadi varga \\
\hline 6. & Abhidhanaratnamala & Swaduskandha \\
\hline 7. & Madanapala Nighantu & Saka varga \\
\hline 8. & Raja Nighantu & Satahvadi varga \\
\hline 9. & Kaiyyadeva Nighantu & Oushadhi varga \\
\hline 10. & Bhavaprakasha Nighantu & Saka varga \\
\hline 11. & Saraswati Nighantu & Ulapadi varga \\
\hline 12. & Saligrama Nighantu & Saka varga \\
\hline 13. & Nighantu Adarsha & Hamsarajadi varga \\
\hline 14. & Priya Nighantu & Satapushpadi varga \\
\hline
\end{tabular}

\subsection{SYNONYMS AS PER DIFFERENT CLASSICS ${ }^{[6,7,8,9,10,11,12,13,14,15,17]}$}

Morphological identity of Sunishannaka is well explained by its synonyms. Nighantus are the main source of synonyms, of which Raja Nighantu enlists the maximum number of synonyms. Synonyms mentioned in various texts are tabulated below.

Table: 2 Difference of opinion regarding synonyms of Sunishannaka

\begin{tabular}{|l|l|l|l|l|l|l|l|l|l|l|l|l|}
\hline $\begin{array}{l}\text { Sl. } \\
\text { N }\end{array}$ & Synonyms & A.H & D.N & So.N & ARM & M.N & R.N & K.N & BP.N & Sa.N & S.N & P.N \\
\hline 1. & Balada & - & - & - & - & + & - & - & - & - & - & - \\
\hline 2. & Baphram & - & - & - & - & - & + & - & - & - & - & - \\
\hline 3. & Cangeri sadrasah dala & - & - & - & - & - & - & - & - & - & - & + \\
\hline 4. & Cangeri sadrasah patra & + & - & - & - & - & - & - & + & - & - & - \\
\hline 5. & Caturdala & + & - & - & + & - & - & - & + & - & - & - \\
\hline 6. & Catushpatri & - & - & - & - & - & - & + & + & - & - & + \\
\hline 7. & Karkatapriya & - & - & - & + & - & - & - & - & - & - & - \\
\hline 8. & Katuka & - & - & - & - & - & + & - & - & - & - & - \\
\hline 9. & Kukkuta & - & + & - & - & - & - & + & + & + & - & - \\
\hline 10. & Kutaja & - & - & + & - & - & - & - & - & - & - & - \\
\hline 11. & Parnakah & - & - & - & - & - & - & - & + & + & - & - \\
\hline 12. & Sikhi & - & + & + & - & - & + & + & + & + & - & - \\
\hline 13. & Sitivaarah & - & + & + & - & - & - & + & - & + & - & - \\
\hline 14. & Sitivarah & - & + & + & - & - & + & - & + & + & - & - \\
\hline 15. & Srivarakah & - & + & + & - & - & + & + & + & + & - & - \\
\hline 16. & Sucidala & - & - & - & - & - & + & - & - & - & - & - \\
\hline 17. & Sucipatrah & - & + & + & - & - & + & + & + & + & - & - \\
\hline 18. & Sucyahvah & - & + & - & - & - & + & - & - & - & - & - \\
\hline 19. & Suksmapatra & - & - & - & + & - & - & - & - & - & - & - \\
\hline 20. & Sulya & - & - & + & - & - & - & - & - & - & - & - \\
\hline 21. & Sunishanna & - & - & - & - & - & - & - & - & - & + & - \\
\hline
\end{tabular}




\begin{tabular}{|l|l|l|l|l|l|l|l|l|l|l|l|l|}
\hline 22. & Sunisannakam & - & + & - & - & - & - & - & - & - & + & + \\
\hline 23. & Sushena & - & - & - & - & + & - & - & - & - & - & - \\
\hline 24. & Svastika & + & + & + & + & + & + & + & + & + & + & + \\
\hline 25. & Swetaambara & - & - & - & - & - & + & - & - & - & - & - \\
\hline 26. & Tilaparnika & - & - & - & - & + & - & - & - & - & - & - \\
\hline 27. & Upodakam & - & - & - & + & - & - & - & - & - & - & - \\
\hline 28. & Upodi & - & - & - & + & - & - & - & - & - & - & - \\
\hline 29. & Vayasi & - & - & - & + & - & - & - & - & - & - & - \\
\hline 30. & Vitunna & - & - & - & - & - & - & + & - & - & + & - \\
\hline
\end{tabular}

\subsection{INTERPRETATION OF SYNONYMS}

Along with drug identification, synonyms gives a comprehensive idea regarding the habitat, pharmacological properties and activities of the plant.

Based on morphology: ${ }^{[18,19]}$

1. Cangeri sadrasah patra - Plant has leaves like Changeri plant

2. Cadurdala - Leaves are four in number

3. Catuspatri - Plant with four leaves

4. Svastika - Represents a form of wheel of Solar Visnu, consisting of

four spokes crossing each other at right angles.

5. Sushena - Having beautiful clusters.

Based on habitat:
6. Upodakam
- That which is seen near to water.

Based on Properties and Action:
7. Sushni
- Plant has sleep inducing property
8. Vitunna
- Pierced or torn

1.6 PHARMACOLOGICAL PROPERTIES ACCORDING TO CLASSICS ${ }^{[7,8,11,12,13,17,20]}$

Rasa of Sunishannaka is descibed as madhura and kashaya in majority of the nighantus but Raja Nighantu and Dhanwanthari Nighantu has attributed only kashaya rasa to the drug. Laghu and Ruksha guna of the drug is being highlighted by most of the nighantus. Regarding the virya of Sunishannaka, all have quoted Seeta virya except Raja Nighantu. Vipaka of the drug is not given in any of the nighantus except in Nighantu Adarsha as katu vipaka. Prabhava have not been mentioned anywhere in the nighantus.

Table: 3 Difference of opinion regarding Rasa panchaka of Sunishannaka

\begin{tabular}{|l|l|l|l|l|}
\hline Nighantus & Rasa & Guna & Virya & Vipaka \\
\hline D.N & Kashaya & - & Sita & - \\
\hline So.N & Kashaya & Laghu & - & - \\
\hline R.N & $\begin{array}{l}\text { Madhura } \\
\text { Kashaya }\end{array}$ & $\begin{array}{l}\text { Laghu } \\
\text { Ruksha }\end{array}$ & Usna & - \\
\hline K.N & Madhura & $\begin{array}{l}\text { Laghu } \\
\text { Ruksha }\end{array}$ & Hima & - \\
\hline BPN & - & Sita & - \\
\hline N.A & Kashaya & - & Sita & Katu \\
\hline P.N & $\begin{array}{l}\text { Swadu } \\
\text { Kashaya }\end{array}$ & - & \\
\hline
\end{tabular}

\subsection{ACTION ON DOSHA AS PER AYURVEDIC TEXTS ${ }^{[6,4,8,10,11,12,13,15,20,17]}$}

The dosha samanatva of Sunishannaka is tridoshaharatva as per all acharyas.

Table: 4 Action of Sunishannaka on Dosha as per Ayurvedic texts

\begin{tabular}{|l|c|c|c|c|c|c|c|c|c|c|}
\hline Doshasamana & A.H & C.S & So.N & R.N & M.P.N & K.N & B.P.N & S.A & N.A & P.N \\
\hline Tridoshahara & + & + & + & + & + & + & + & + & + & - \\
\hline Manodoshapahara & - & - & - & - & - & - & - & - & - & + \\
\hline
\end{tabular}




\subsection{KARMA OF SUNISHANNAKA AS PER CLASSICS ${ }^{[7,8,10,12,13,11,17,6]}$}

Nighantus and Samhitas provides the basic informations regarding the actions of Sunishannaka which mainly includes its vrishya, grahi, vastisudhikara, agnikara, deepana, hridya, rasayana, rucya and medhya karmas.

Table 5: Karma of Sunishannaka as per different Ayurvedic texts

\begin{tabular}{|l|l|l|l|l|l|l|l|l|}
\hline Karma & D.N & So.N & M.P.N & K.N & B.P.N & R.N & $P . N$ & A.H \\
\hline Vrishya & + & + & - & + & + & - & + & + \\
\hline Grahi & + & + & + & + & + & - & - & - \\
\hline Samgrahi & + & - & - & - & - & + & + & - \\
\hline Vastisudhikara & + & - & - & - & - & - & - & - \\
\hline Agnikrit & + & + & - & - & - & - & - & + \\
\hline Deepana & - & - & - & + & + & - & - & - \\
\hline Avidahi & - & - & - & + & + & - & - & - \\
\hline Hridya & - & - & - & + & - & - & - & - \\
\hline Rasayana & - & - & - & - & - & + & - & - \\
\hline Rucya & - & - & - & - & + & - & - & - \\
\hline Medhya & - & - & - & - & - & - & + & - \\
\hline
\end{tabular}

\subsection{THERAPEUTIC INDICATION}

Sunishannaka has been mentioned in the chikitsa aspects of many diseases like hridroga, pleeha, gulma, arsha, meha, jwara, swasa, kushta, aruchi, bhrama, daha etc. The opinion of different acharyas are tabulated below.

Table: 6 Difference of opinion regarding therapeutic indications in Nighantus.

\begin{tabular}{|l|l|l|l|l|l|l|l|}
\hline Indication & D.N & M.P.N & K.N & B.P.N & $\begin{array}{l}\text { Guna } \\
\text { ratnamala }\end{array}$ & R.N & P.N \\
\hline Hridroga & + & - & - & - & - & - & - \\
\hline Pleeha & + & - & - & - & - & - & - \\
\hline Gulma & + & - & - & - & - & - & - \\
\hline Arsha & + & - & - & - & - & - & - \\
\hline Meha & & + & + & + & + & - & - \\
\hline Jwara & - & - & + & + & + & - & + \\
\hline Swasa & - & - & + & + & + & - & - \\
\hline Kushta & - & - & + & + & + & - & - \\
\hline Aruchi & - & - & + & + & - & - & - \\
\hline Bhrama & - & - & + & + & - & - & - \\
\hline Bhranti & - & - & - & - & - & - & + \\
\hline Daha & - & - & - & - & - \\
\hline
\end{tabular}

\subsection{PRAYOJYA ANGA [PART USED] $]^{[21]}$}

Whole plant

1.11 MATRA[DOSAGE] $]^{[22]}$

Juice -10 to $20 \mathrm{ml}$

1.12 AMAYIKA PRAYOGA ${ }^{[23,24,25,29,30,31]}$

> Vataja kasa - Saka of Sunishannaka as a wholesome diet is adviced.

$>$ Urustambha - Sunishannaka cooked in water and oil without adding salt given as a diet.

$>$ Vatarakta - Sunishannaka cooked with ghrutha and mamsarasa.

$>$ Visha - Advised as a wholesome green leafy vegetable.

D Raktapitta - Used as Saka ahara, after frying in ghrutha.

> Vrana - lepa with Sunishannaka patra kalka

> Mutrakrichra - Intake of Sunishannaka beeja choorna along with takra.

> Asthisrava - Intake of Sunishannaka along with milk.

- Intake of Mukkudi with Sunishannaka and Musta

- Intake of Sunishannaka with sugar for 4 days cures all types of srava. 
Jwara - Kashaya prepared with Sunishannaka, venupatra, sunti, musta, and guduchi cures Sannipatha jwara within 3days.

\subsection{FORMULATIONS OF SUNISHANNAKA}

Ghruta kalpana

Sunishannakacangeri ghruta.

Avakpushpadyam ghrutha ${ }^{[32]}$

\section{Kashaya kalpana}

Pathram venuvaadikashayam. [Sahasrayogam, Jwara chikitsa]

\section{Conclusion:-}

Hence it can be concluded that the drug Sunishannaka mentioned in Samhitas and Nighantus have the properties of tridoshaghnata, vrishya, grahi, vastisudhikara, agnikara, deepana, hridya, rasayana, rucya and medhya karmas. The rasa panchakas mentioned in Nighantus substantiate its clinical uses. So the drug is having a high potential therapeutically, which has to be proved clinically.

\section{References:-}

1. Sastri. J L N. Dravyaguna vijnana, Knowledge on vedic herbs, controversial herbs and ignored medicinal plants Vol V, Chaukhamba Orientalia; Varanasi: 2011; Page no:86.

2. Tharanatha tarka vachaspati Bhattacharya, Vachaspatyam, 6th volume, Choukhambha ayurveda prathishtan, 2006th edition, Page no 4505.

3. Moosad Parameswaram T C Vachaspathi, Amarakosam, Reprint 1959, Kottayam, Sahithya Pravarthaka Cooperative Society Ltd.

4. Sharma R K and Dash B. Charaka Samhitha: English translation and critical exposition based on Chakrapanidatta's Ayurvedadipika. Chowkamba Sanskrit Series, Varanasi, 2016; Vol 1; p: 509-510.

5. Sreekantha Murthy. Translator, Illustrated Susruta Samhita, Reprint 2014, Varanasi, Chaukambha Orientalia, Vol 1, p: 301.

6. Sreekantha Murthy. Translator, Ashtanga Hrudaya of Vagbhata, Reprint 2013, Varanasi, Chaukambha Orientalia, Vol:1, p:89.

7. Kamat. S.D, Studies on Medicinal Plants and Drugs in Dhanvantari Nighantu, 2002, Delhi, Choukhambha Sanskrit Pratishthan, page no: 56-57

8. Dwivedi R.R ,Editor, Sodhala nighantu by Acharya Sodhala ,Guna samgraha part, 1st edition, Choukhambha Krishnadas Office, 2009, page no: 40.

9. Sharma P.V, Abhidhana Ratnamala (Shadrasa Nighantu), Reprint Edition, Varanasi, Choukhambha orientalia, 2013, Page no: 9

10. Tripathi Harihara Prasad, Editor, Madanapala Nighantu by Madanapala with Hari hindi commentary, First edition, Varanasi, Choukhambha Krishnadas Academy, 2009, Page no:177.

11. Tripathi Indradev, Editor, Raja Nighantu of Pandit Narahari, Fourth edition, Varanasi, Choukhambha Krishnadas Academy, 2006, p: 71

12. Sharma P V. \& Sharma Guru Prasad, Editor \& Translator, Kaiyadeva Nighantu, Reprint 2013, Varanasi, Choukambha Orientalia, p:127.

13. Pandey G S. Editor. Chunekar K C. Commentator, Bhavaprakasha Nighantu of Bhavamisra, Reprint 2013, Varanasi, Choukambha Bharati Academy, p:661.

14. Kamat S.D. Editor. Studies on medicinal plants and drugs in Saraswati Nighantu, First edition, Delhi, Chaukhamba Sanskrit Pratishthan, p:52.

15. Saligrama. Saligrama Nigantu- Bhooshanam. Bombay: Khemraj Srikrishnadas Prakasan; 2006: Part 7 \&8; pg no. 655 .

16. Vaidya G. Bapalala, Nighantu Adarsha, Volume 2 Reprint 2016, Varanasi, Chaukhambha Bharati Academy, p:774.

17. Sharma P V, Priya Nighantu, Edition 2004, Varanasi, Chaukhamba Surbharati Prakashan, p:98.

18. Vaidya Bapalal. Some Controversial Drugs in Indian Medicine, Chaukambha Orientalia, Reprint: 2014; p: 212213.

19. Sir Monnier \& Williams. Sanskrit English dictionary, 16th reprint, 2011, Delhi, Motilal Barasidass Publishers Private Limited, Vol:1; p:324. 
20. Vaidya G. Bapalala, Nighantu Adarsha, Volume 2 Reprint 2016, Varanasi, Chaukhambha Bharati Academy, p:774.

21. Indian medicinal plants a compendium of 500species, Edited by Arya Vaidya Sala, Kottakkal, Orient Longman Ltd, Madras, Vol 4, p: 5.

22. Pandey Gyanendra, Dravyaguna vijnana, Chowkhamba Krishnadas Academy, Varanasi; Reprint: 2004; Vol 3; p: 546.

23. Sharma R K and Dash B. Charaka Samhitha: English translation and critical exposition based on Chakrapanidatta's Ayurvedadipika. Chowkamba Sanskrit Series, Varanasi, 2016; Vol IV; p:176-177.

24. Sharma R K and Dash B. Charaka Samhitha: English translation and critical exposition based on Chakrapanidatta's Ayurvedadipika. Chowkamba Sanskrit Series, Varanasi, 2013; Vol V; p: 102-103.

25. Sharma R K and Dash B. Charaka Samhitha: English translation and critical exposition based on Chakrapanidatta's Ayurvedadipika. Chowkamba Sanskrit Series, Varanasi, 2016; Vol IV; p: 378.

26. Vaidya Yadavaji Trikamji, Editor, Susrutha samhitha of Susrutha with Nibadhasamgraha commentary of Dalhana, uthara sthana, Ch 45, Ver 16, Choukhambha Sanskrit Samsthan, p: 735.

27. Sreeman Nambootiri. Editor, Chikitsamanjari, Kodungallor, Vidyarambham Publications, 7th edition, Reprint:2005, p:62,95.

28. Sreeman Nambootiri. Editor, Chikitsamanjari, Kodungallor, Vidyarambham Publications, 7th edition, Reprint:2005, p:109.

29. Sreeman Nambootiri. Editor, Yogamrutam, Kodungallor, Vidyarambham Publishers, $3^{\text {rd }}$ edition, Reprint 2004, $\mathrm{p}: 140$

30. Sreeman Nambootiri. Editor, Yogamrutam, Kodungallor, Vidyarambham Publishers, $3^{\text {rd }}$ edition, Reprint 2004 , $\mathrm{p}: 141$

31. Krishnanvaidyan K V, Gopalapillai S. Sahasrayogam: Sujanapriyavyaknam.32nd Edition..Alapuzha: Vidyarambham publishers ; 2013, P: 30

32. Ganga sahaya Pandeya, Editor, Gadanigraha of Sri Vaidya Sodhala with the'Vidyotini' Hindi commentary, Chaukhambha Sanskrit Sansthan, edition 2011,part 2, p: 69. 